Sciences

Vol. 06, No. 02, pp. 77-85, June 2013

\title{
GENERATION OF ENTANGLED PHOTONS BY A SHORT COHERENCE LENGTH VIOLET DIODE LASER
}

\author{
Ra'ad Subhi Abbood $^{1}$, Ahmed I. Khaleel ${ }^{2}$, Shelan Kh. Tawfeeq ${ }^{3}$ \\ 1,2,3 Institute of Laser for Postgraduate Studies, Baghdad University \\ Email: raad.1990.1990@gmail.com¹,infoahmed77@yahoo.com², sheelanf@yahoo.com³ \\ (Received:27/6/2011; Accepted:16/9/2012)
}

\begin{abstract}
Pumping a BBO crystal by a violet diode laser with a wavelength of (405 $\mathrm{nm})$ output power of $(24 \mathrm{~mW})$ and a line width of $(3 \mathrm{~nm})$ was employed to generate entangled photons with a wavelength of $810 \mathrm{~nm}$ by achieving type II phase matching conditions.

The coincidence count rate obtained in this experiment was in the range of (18000) counts/s. Two BBO crystals with different thicknesses of (4 mm and $2 \mathrm{~mm})$ were tested, where maximum count rates of about (18000) counts/s was obtained with a $(5 * 5 * 2) \mathrm{mm}$ BBO crystal where the short coherence time for the pumping source was tolerated by using shorter BBO crystals. Also, the effect of compensating crystal on the walk-off effect was studied. The coincidence count rates were increased by using these crystals when their optic axes were fixed in the opposite direction relative to the optic axis of the original BBO crystal.
\end{abstract}

Keywords: Entanglement, EPR, spontaneous parametric down conversion.

\section{INTRODUCTION}

Entanglement is one of the most remarkable features of quantum mechanics which can not be understood in the context of classical physics. It has been shown that entanglement can exist in various physical systems and play a role in quantum phenomena. Moreover, its properties can be used as a resource for quantum information technologies such as quantum computing, quantum cryptography, and quantum communication ${ }^{(1)}$.

Entangled states have the fascinating property that, even if the individual qubits are separated by great distances, one cannot describe their behaviors independently. The two qubits must still be treated as single quantum system $^{(2)}$. 
Whenever a polarization measurement is performed on the photon of the pair, automatically the other photon is projected in a definite polarization state, so that we can endorse that one of legitimate users of entanglement plays the role of triggering the second user measurements ${ }^{(3)}$.

There are four maximally entangled two qubit states which can be used for quantum cryptography, known as the Bell States. They have the following form using the polarization of photons ${ }^{(4)}$.

$$
\begin{aligned}
& \left|\psi^{+}\right\rangle=\frac{1}{\sqrt{2}}(|H V\rangle+|V H\rangle) \\
& \left|\psi^{-}\right\rangle=\frac{1}{\sqrt{2}}(|H V\rangle-|V H\rangle) \\
& \left|\psi^{+}\right\rangle=\frac{1}{\sqrt{2}}(|H H\rangle+|V V\rangle) \\
& \left|\psi^{-}\right\rangle=\frac{1}{\sqrt{2}}(|H H\rangle-|V V\rangle)
\end{aligned}
$$

These equations form a complete orthonormal basis for all polarization states of a two photon system.

Entangled photons may be generated by SPDC using BBO crystals. Due to the birefringence of the BBO crystal the propagation directions and velocities of horizontally and vertically polarized light are slightly different as they travel through the crystal. This leads to a transverse walk-off (spatial displacement) and longitudinal walk-off (temporal displacement) of the horizontally polarized photons from the vertically polarized photons, thus the photons can be distinguished by their position and time-correlation relative to one another. While the walk-off effect cannot be truly compensated, the distinguish ability of the photons can be washed out by the use of compensator BBO crystals. First, the downconverted photons have their polarizations rotated by $90^{\circ}$ using a half wave plate so that $\mathrm{H}$ is exchanged with $\mathrm{V}$ and vice versa. Each photon is then passed through a compensator BBO crystal which is half as thick as the original crystal that performs down-conversion. The walkoff effects are reversed by half, which consequently erases all temporal distinguish ability and reduces the effects of the transverse walk-off. The temporal compensation for walk off effect can be seen in Figure(1) ${ }^{(4)}$.

The maximal walk-off effect occurs for pairs created near the entrance face, which consequently acquire a relative time delay ${ }^{(5)}$,

$\delta \tau=L\left(1 / u_{s}-1 / u_{\alpha}\right)$

where,

$\mathrm{L}$ is the crystal length. 
$u_{o}, u_{\vartheta}$ are the ordinary and extraordinary group velocities.

and a relative lateral displacement:

$d=L \tan \rho$

where,

$\rho$ is the angle between the ordinary and extraordinary beams inside the crystal.

The purity and visibility of the generated entangled state depend on the crystal's length and the coherence time of the pump ${ }^{(6)}$.

If:

1- $\delta \tau \gg \tau_{a}$ there is an incoherent superposition of random phases then the entangled photons become distinguishable, i.e., entanglement will be washed out otherwise there exists a coherent superposition ${ }^{(6)}$.

$2-\delta \tau<\tau_{v}$ the generated entangled state is characterized by high purity.

3- $\delta \tau \stackrel{M}{=} \tau_{\sigma}$, the purity is degraded.

where,

$\tau_{\sigma}$ is the coherence time of the pump laser.

As the crystal length increases, $\delta \tau$ increases and it may become longer than $\tau_{\varepsilon}$ which means canceling entanglement ${ }^{(6)}$.

The optical anisotropy of the crystal which causes the difference in crystal transit time has no significant effect on the entanglement when the incident pump photons have a negligible bandwidth. As the pump spectral profile become broader, the effect of optical anisotropy of the crystal becomes significant ${ }^{(7)}$.

Finding the appropriate conditions for a particular parametric process is called phase matching. Dispersion relations, birefringence, and temperature dependence of refractive indices allow phase matching to be achieved by choosing appropriate frequencies, angles, and temperatures ${ }^{(8)}$.

Equation 13 shows the relation between crystal cut, pump direction and phase matching $\operatorname{angle}^{(8)}$.

$\frac{1}{n_{g}\left(\theta_{p m}\right)^{2}}+\frac{\sin ^{2} \theta_{p m}}{n_{g}^{2}}=\frac{\cos ^{2} \theta_{p m}}{n_{0}^{2}}$

where,

$\theta_{p m i}$ is the angle between the optic axis and propagation direction.

$n_{w}$ is the extraordinary refractive index. 
$n_{o}$ is the ordinary refractive index.

With type-II phase matching, the two cones are tangent to one another on exactly one line which is the pump beam direction. If the pump beam angle $\theta_{p m}$ as shown in Figure 2 is decreased, the two cones will separate from each other entirely as shown. If the angle is increased, the two cones tilt toward the pump beam causing an intersection along two lines. Along the two directions, where the cones overlap, the light can be essentially described by an entangled state ${ }^{(5)}$.

\section{THE EXPERIMENTAL SETUP}

In this set up is a single-mode $\mathrm{CW}$ laser diode of wavelength $405 \mathrm{~nm}$ with an output power of $24 \mathrm{~mW}$ is focused to the desired beam diameter size $(400 \mu \mathrm{m})$ with a spherical lens with a focal length of $50 \mathrm{~mm}$.The elliptical profile of the pump beam is compensated using an additional cylindrical lens with focal length of $(25 \mathrm{~mm})$. The $(5 * 5 * 4) \mathrm{mm}$ and $(5 * 5 * 2) \mathrm{mm}$ BBO ( $\beta-\mathrm{BaB} 204)$ crystals used for generation process, cut for type-II phase matching, were placed at the minimum diameter position of the pump beam. For degenerate emission the intersection lines of the emission cones form an opening angle of $6^{\circ}$ and the tangents in the crossing points are perpendicular to each other. Two photon counting modules from micro photon devices (MPD) were used for the detection of the entangled photons at $810 \mathrm{~nm}$. The detection efficiency of these photon counting modules at $810 \mathrm{~nm}$ is approximately equal to $15 \%$. In front of each detection module an IR filter with center wavelength of $810 \mathrm{~nm}$ was fixed in order to distinguish the photons generated at $810 \mathrm{~nm}$ from any background photons.

Two BBO crystals with dimensions of $(5 * 5 * 2) \mathrm{mm}$ were each fixed in the path of the generated entangled photons in order to compensate for the walk-off effect.

In order to distinguish the entangled photons pairs, the generated photons at $810 \mathrm{~nm}$ were detected within $20 \mathrm{~ns}$ window. This was achieved by building an electronic circuit for this purpose as shown in Figure(3).

Counter circuit was also built in order to count the number of photons detected by each detection module and the number of coincidence entangled photons pairs per a time window of one second.

Figure(4) shows the set up for generation of entangled photons by parametric down conversion process. 


\section{RESULTS AND DISCUSSION}

The results were taken for using two BBO crystals for generation of the entangled photons with dimension of $(5 * 5 * 4) \mathrm{mm}$ and $(5 * 5 * 2) \mathrm{mm}$ in order to record the effect of the crystal thickness on the rate of generation of the entangled photons. Also the effect of using compensating crystals was studied for both cases of fixing these crystals optic axis at the same direction as that of the crystal used for generation entangled photons, and fixing the compensating crystals optic axis in opposite direction. The results are listed in Table(1).

Maximum coincidence count rates was obtained when the crystal was rotated $\left(11^{\circ}\right)$, tilted and moved along the z-direction $(9 \mathrm{~cm})$. Meaning that, achieving the best phase matching conditions. Also the detection modules were isolated from the background light by using cartoon boxes in order to overcome the low detection efficiency at $810 \mathrm{~nm}$.

From the results listed in Table(1), maximum coincidence count rate (18000 counts/s) was obtained with using $(5 * 5 * 2) \mathrm{mm}$ BBO crystal for generation of the entangled photons because shorter thickness of the crystal reduce the delay, $\boldsymbol{\delta} \boldsymbol{\tau}$, between the generated photons to tolerate for the short coherence time of our violet diode laser due to the long line width for our pumping source, $3 \mathrm{~nm}$ giving a coherence length of $\left(5.46 \times 10^{-5} \mathrm{~m}\right)$ compared with ${ }^{(17)}$ and ${ }^{(13)}$, where the coherence length equals to $(3 \mathrm{~m})$ and $\left(1.632 \times 10^{-4} \mathrm{~m}\right)$ respectively. This coincidence count rate was obtained with the BBO compensating crystals optic axis aligned in the opposite direction with that of the original crystal i.e, reducing the walk off effect.

\section{CONCLUSIONS}

Accepted results for SPDC process for generating entangled photons can be obtained by tolerating some poor characteristics of the pumping source, violet diode laser with a line width of $3 \mathrm{~nm}$, and low detection efficiency for the detection modules $(15 \%$ at 810 $\mathrm{nm}$ ) by using short BBO crystals for generating of the entangled photons, meeting the best phase matching conditions as possible, using compensating crystals and isolating the detection modules from the background light.

Better results can be obtained with shorter BBO crystals and higher detection efficiency for the detector module at $810 \mathrm{~nm}$.

\section{REFERENCES}

1- S.W. Lee, H. Jeong, and D. Jaksch ,(2009), " Witnessing entanglement in phase space using inefficient detectors" quanta-ph,3,p1. 
2- E. Waks, (2003), "Quantum information processing with non-classical light" Ph.D. Thesis, Stanford University, p30.

3- S. Castelletto, I. P. Degiovanni, M. L. Rastello, (2002), " Effects of Experimental limits in Quantum Cryptography Systems based on polarization entangled photons",p3.

4- C. Erven, (2007), " On Free Space Quantum Key Distribution and its Implementation with a Polarization-Entangled Parametric Down Conversion Source" MSC.Thesis. University of Waterloo, Ontario, Canada, p3.

5- P.G. Kwait, K. Mattle, H. Wienfurter, and A. Zielinger, A. V. Sergienko and Y. Shih, (1995), "New high intensity of polarization entangled photon pairs"Physical Review Letter,75,No.24,p2.

6- S. Cialdi, F. Castelli, I. Boscolo, and M. G. A. Paris, (2008), " Generation of entangled photon pairs using small coherence-time continuous wave pump lasers", Applied Optics , 47, No.11, p3.

7- D. Erenso, (2009), "Pump Spectral Bandwidth, Birefringence, and Entanglement in Type-II Parametric Down Conversion", Research Letters in Optics, p 3,4.

8- B. A. Betchart, (2004), "A Test of Bell's Inequality for the Undergraduate Laboratory" MSC.thesis Department of Physics and Astronomy, Oberlin College, p 15,16.

9- C. Kurtsiefer, M. Oberparleiter, and H.Weinfurter, (2001), "Generation of correlated photon pairs in type-II parametric down conversion - revisited", p2.

Table (1): Coincidence count rate for using $(5 * 5 * 4) \mathrm{mm}$ and $(5 * 5 * 2) \mathrm{mm}$ crystals with $(5 * 5 * 2) \mathrm{mm}$ compensating crystals.

\begin{tabular}{|c|c|c|c|}
\cline { 2 - 4 } \multicolumn{1}{c|}{} & $\begin{array}{c}\text { Without } \\
\text { compensating } \\
\text { crystal }\end{array}$ & $\begin{array}{c}\text { With compensating } \\
\text { crystal axis at the } \\
\text { same direction of } \\
\text { the BBO crystal }\end{array}$ & $\begin{array}{c}\text { With compensating } \\
\text { crystal axis at the } \\
\text { opposite direction of } \\
\text { the BBO crystal }\end{array}$ \\
\hline$(5 * 5 * 4) \mathrm{mm}$ & 10000 & 7000 & 11000 \\
\hline$(5 * 5 * 2) \mathrm{mm}$ & 17000 & 15000 & 18000 \\
\hline
\end{tabular}




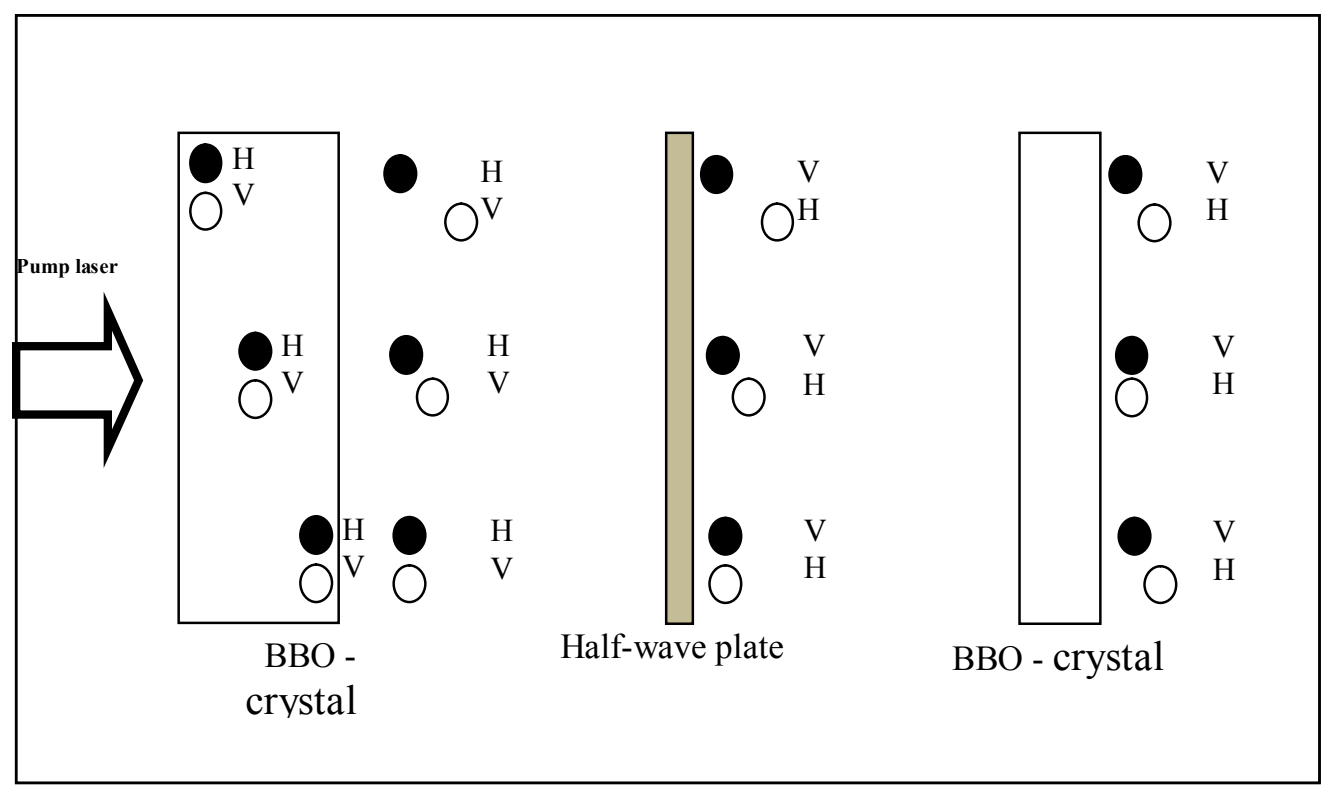

Fig.(1): Longitudinal walk-off of the horizontally polarized photon from the vertically polarized photon occurs due to birefringence of the BBO crystal.

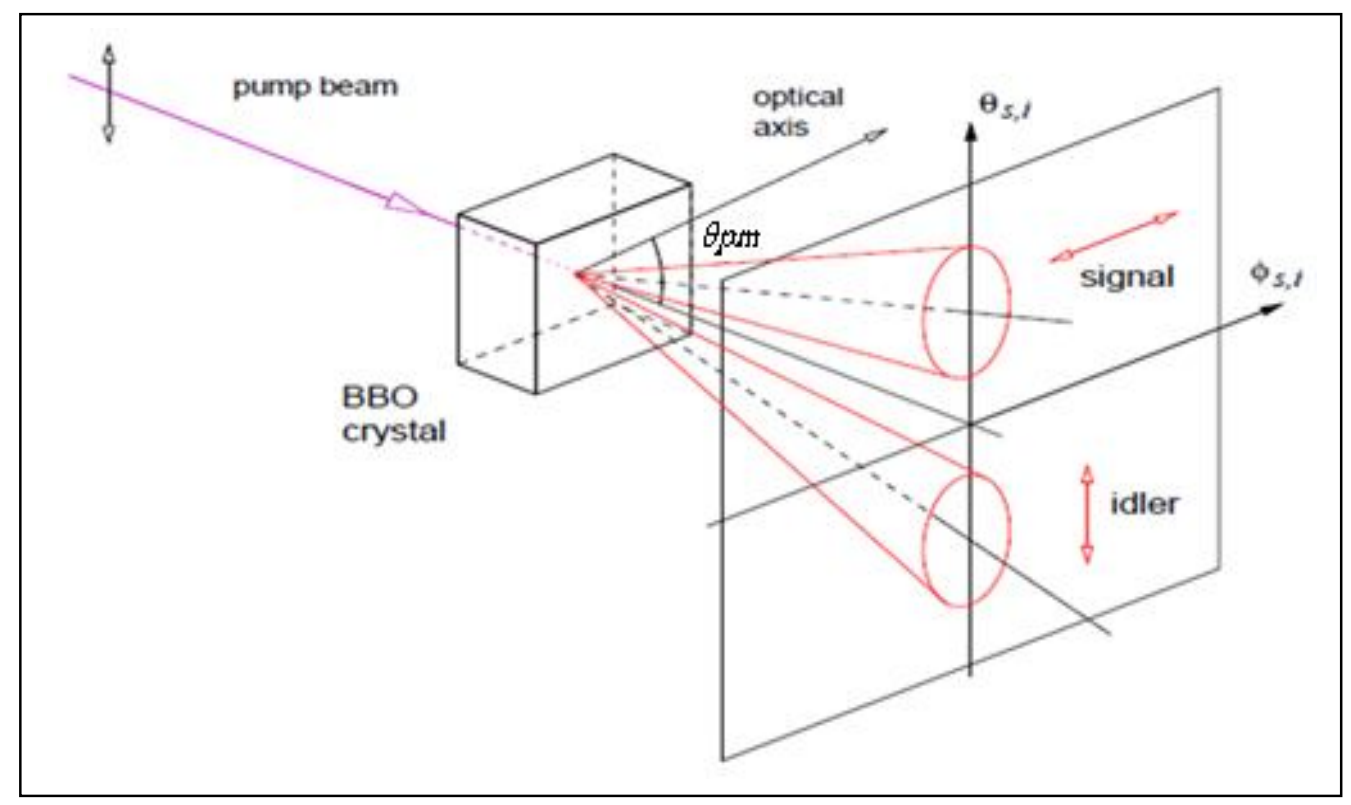

Fig. (2): Type II phase matching entanglement shows the beams corresponding angles ${ }^{(9)}$. 


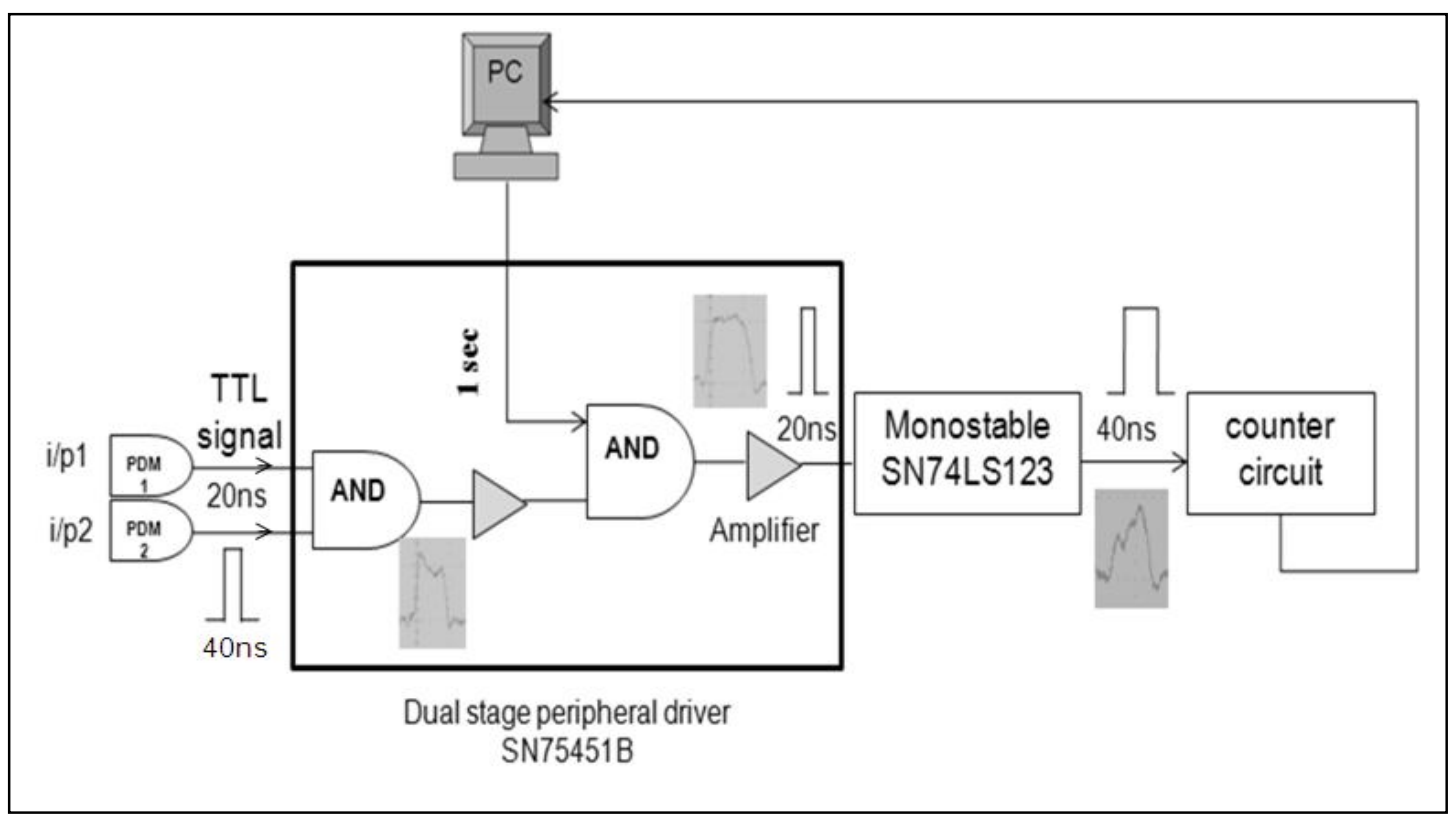

Fig.( 3): Schematic diagram of the coincidence circuit.

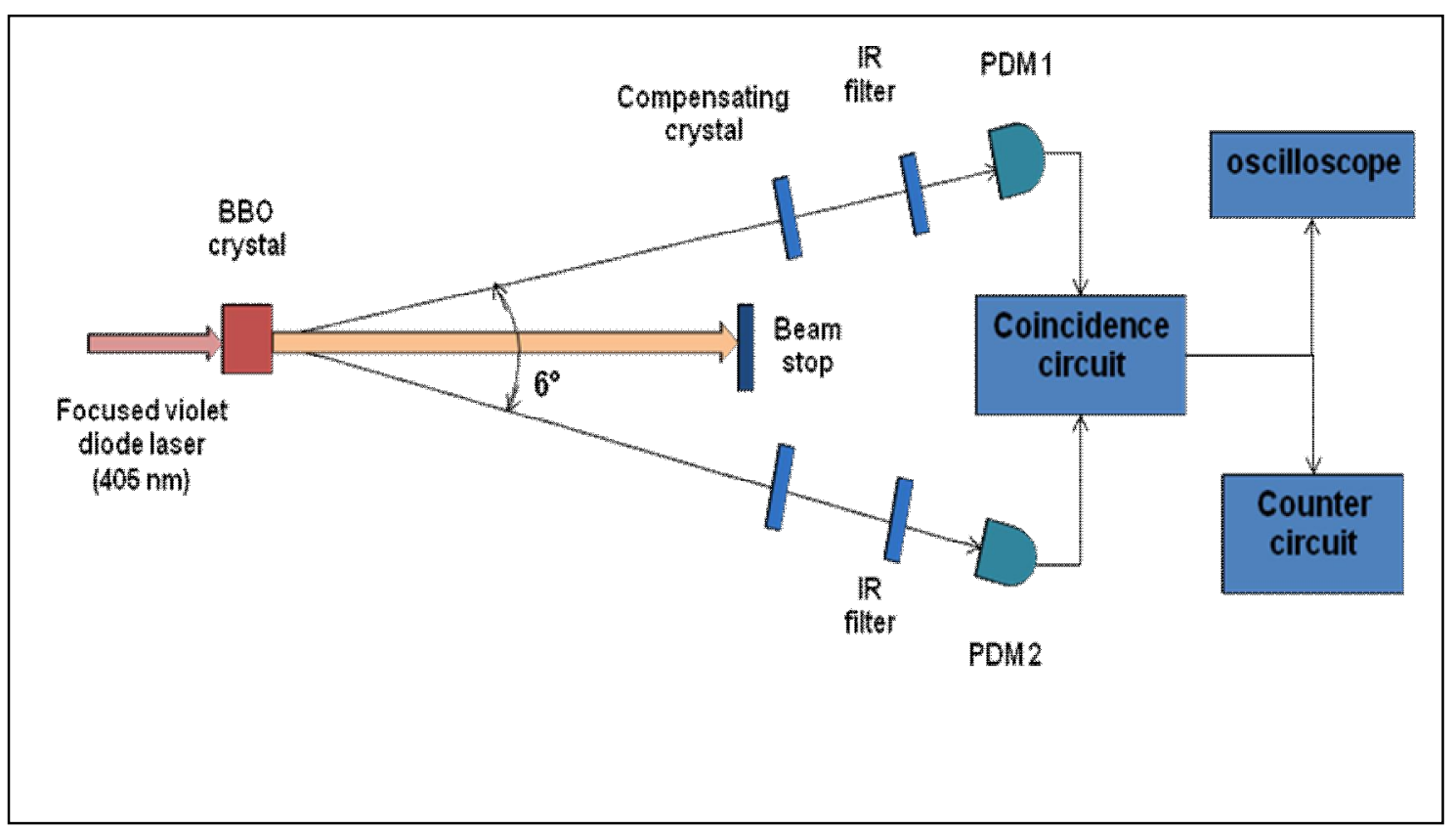

Fig.(4): The schematic diagram for the complete setup. 


\section{توليد الفوتونات المتشابكة بواسطة ليزر ثنائي بنفسجي ذو طول تثاكه قصير}

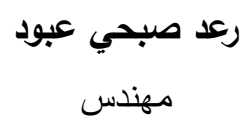

\author{
احمد إسماعيل خليل \\ مدرس مساعد
}

معهد الليزر - جامعة بغداد
شيلان خسرو توفيق

مدرس دكتورة

إن هذا العمل هو لتوليد الفوتونات المتشـابكة بواسطة عملية التحويل العكسية العشوائية المعلمة. يتم تحقيق هذا

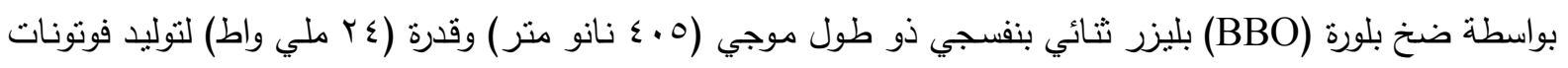

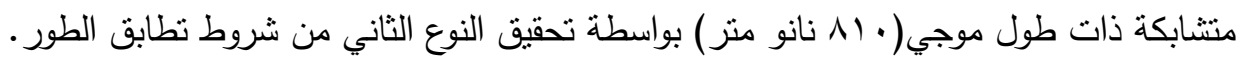

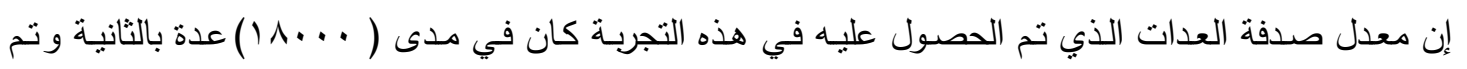

$$
\text { اختبار بلورات (BBO) مع سمك مختلف (4 ملي منر و } 2 \text { ملي متر ). }
$$

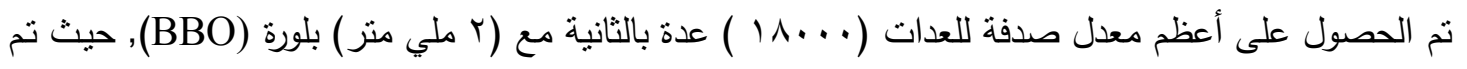

تحمل زمن التشاكه القصبر لمصدر الضخ بواسطة استخدام بلورة (BBO) الأقصر .

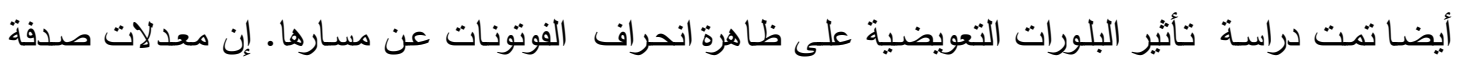

العدات قد تم زيادتها بواسطة استخدام هذه البلورات عندما تكون محاورها البصرية مثبتة بعكس اتجاه المحور البصري للبلورة الأصلية. 